\title{
Levosimendan as Rescue Therapy for Acute Heart Failure in a Patient with Duchenne Muscular Dystrophy
}

\author{
Dorin Sumanaru $^{1} \cdot$ Loic Josseran $^{2,3} \cdot$ Aben Essid $^{1} \cdot$ Blaise Mbieleu $^{1} \cdot$ Isabelle Haegy ${ }^{1}$ Jean Bergounioux ${ }^{1,2,4} \oplus$
}

Received: 10 December 2018 / Accepted: 2 February 2019 / Published online: 22 February 2019

(c) Springer Science+Business Media, LLC, part of Springer Nature 2019

\begin{abstract}
The longer survival of patients with Duchenne muscular dystrophy due to advances in clinical care has increased the incidence of Duchenne muscular dystrophy-associated cardiomyopathy, a nearly consistent feature in the third decade of life. A 26-year-old patient with Duchenne muscular dystrophy experienced severe acute heart failure triggered by pneumonia. Levosimendan was effective in improving heart function.
\end{abstract}

Keywords Duchenne muscular dystrophy $\cdot$ Levosimendan $\cdot$ Acute heart failure

\section{Introduction}

Duchenne muscular dystrophy is an incurable lethal X-linked muscle disease caused by dystrophin deficiency [1]. Skeletal muscle weakness starts to impair mobility at 2-6 years of age, and many patients are wheelchair-bound by early adolescence. Advances in the management of the musculoskeletal and respiratory manifestations of Duchenne muscular dystrophy have improved survival, resulting in an increased incidence of cardiomyopathy, a nearly consistent feature in the third decade [1]. Early diastolic dysfunction and focal fibrosis progress to dilated cardiomyopathy, with heart failure and arrhythmia. Few patients with end-stage Duchenne muscular dystrophy-associated dilated cardiomyopathy are candidates for heart transplantation. Heart failure is now a leading cause of death in patients with Duchenne muscular dystrophy [1] and contributes, together with respiratory

Jean Bergounioux

jean.bergounioux@aphp.fr

1 Pediatric Intensive Care Unit, Hôpital Raymond-Poincaré, APHP, 92380 Garches, France

2 Epidemiology Department, Hôpital Raymond-Poincaré, APHP, 92380 Garches, France

3 Versailles Saint Quentin en Yvelines University, Versailles, France

4 Réanimation et soins intensifs pédiatriques, Hôpital universitaire Raymond Poincaré, AP-HP, 104 Boulevard Raymond Poincaré, 92380 Garches, France insufficiency, to shorten the lifespan by about two-thirds to one-half compared to normal individuals.

Levosimendan is a recently introduced drug that acts via two mechanisms: it sensitizes troponin $\mathrm{C}$ to calcium, in a calcium concentration-dependent manner, thereby improving contractility at a low-energy cost; and it induces vasodilation by opening the ATP-sensitive potassium channels. This combination of inotropic and vasodilating effects increases cardiac output without significantly increasing myocardial oxygen demand [2]. Levosimendan improved the NYHA class in patients with end-stage heart failure [3] and offered a safe and effective alternative to dobutamine dosage escalation in patients with septic myocardial depression [4] or severe low-output heart failure [5].

\section{Case Report}

We used levosimendan in a 26-year-old patient with Duchenne muscular dystrophy-associated cardiomyopathy, in whom an episode of pneumonia triggered severe low-output heart failure. He had a history of surgical spinal fusion for scoliosis at age 14 years, chronic cardiomyopathy since age 17 years (left ventricular ejection fraction $46 \% 2$ months before admission), and tracheotomy with ventilator dependency since age 18 years. At ICU admission, he was started on mechanical ventilation, fluid loading, and continuous intravenous vasopressor therapy. His hemodynamic status and respiratory function improved. However, on hospitalization day 4 , he showed deteriorating myocardial contractility 
with severe segmental dysfunction and a left ventricular ejection fraction of $30 \%$ as estimated by transthoracic echocardiography. Despite increases in the dosages of dobutamine (to $15 \mu \mathrm{g} / \mathrm{kg} / \mathrm{min}$ ) and norepinephrine (to $1 \mu \mathrm{g} / \mathrm{kg}$ / $\mathrm{min}$ ), his haemodynamic status worsened, leading to anuria, oedema, serum lactate elevation $(5 \mathrm{mmol} / \mathrm{L})$, and increased ventilatory support requirements. On hospitalization day 8 , levosimendan was started as a continuous infusion of $0.1 \mu \mathrm{g} / \mathrm{kg} / \mathrm{min}$, with no loading dose given the presence of premature atrial contractions. After $8 \mathrm{~h}$, when one-third of the planned total dose had been given, levosimendan was stopped because of sustained sinus tachycardia at $210 \mathrm{~b} / \mathrm{min}$.

Heart function improved within a few hours after levosimendan initiation and sinus rhythm came back to normal. The ventilatory requirements diminished, urine output returned to normal, and serum levels of both lactate and brain natriuretic peptide declined (Fig. 1). Resolution of the segmental myocardial dysfunction was noted, with uniform left ventricular contraction and an increase in left ventricular ejection fraction to $50 \%$. The pneumonia resolved completely. Vasopressor therapy was discontinued a few days after the period of levosimendan administration. The hemodynamic condition remained stable, with a normal sinus rhythm. However, the patient died suddenly from ventricular fibrillation on day 40 after ICU admission.

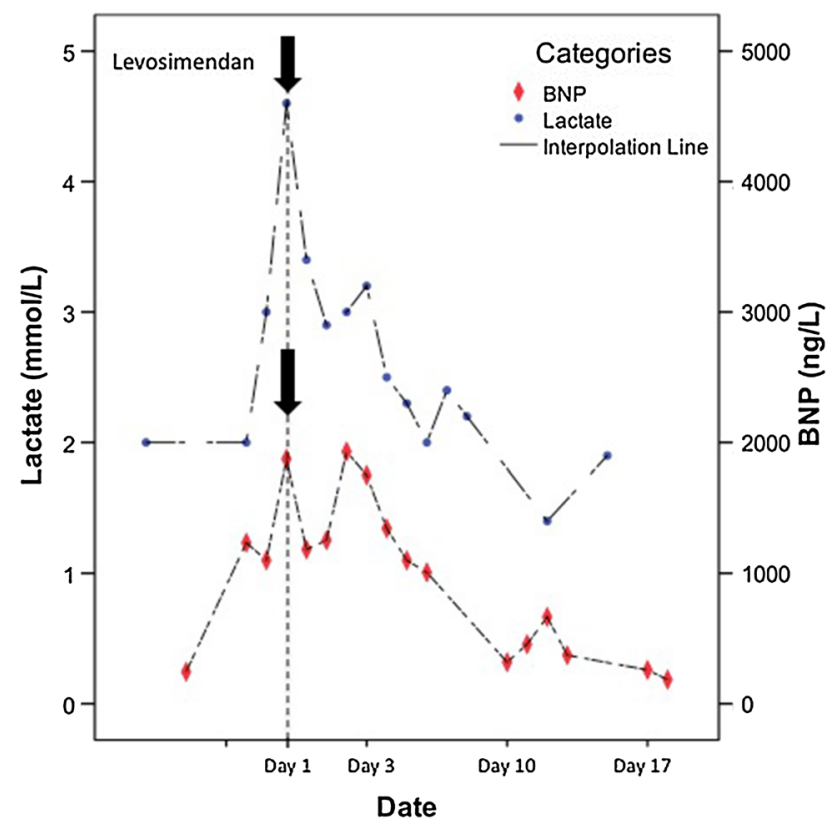

The evolutions of the serum lactate level and the Brain Natriuretic Peptide after Levosimendan

Fig. 1 Serum brain natriuretic peptide and lactate levels starting day 4 after intensive-care-unit admission and before and after levosimendan therapy (considered as Day1). BNP brain natriuretic peptide. $\mathrm{X}$-axis: day after Levosimendan administration

\section{Discussion}

Levosimendan induces a rapid dose-dependent improvement in hemodynamic function in patients with decompensated heart failure. Data from clinical studies suggest that these hemodynamic benefits may translate into symptom alleviation without significantly increasing the frequency of adverse events [5,6]. The steady state is achieved within $5 \mathrm{~h}$ of initiation of a constant-rate intravenous infusion and the elimination half-life is $1 \mathrm{~h}$. In the body, acetylation of levosimendan generates two biologically active metabolites, OR-1896 and OR-1855, whose considerably longer half-lives [7] produce pharmacological effects for 1 week. Our patient received only one-third of the full levosimendan dose, as he developed sinus tachycardia at $210 \mathrm{~b} / \mathrm{min}$. Nevertheless, his symptoms improved rapidly. As shown in Fig. 1, this effect was sustained and the left ventricular ejection fraction remained stable until the fatal episode of arrhythmia. A remarkable event was the correction of segmental myocardial dysfunction within $6 \mathrm{~h}$ after the end of the infusion. Transthoracic echocardiography showed restoration of uniform left ventricular contraction, which greatly contributed to increase the cardiac output.

There is no evidence that short-term levosimendan therapy used to treat heart failure increases the risk of cardiac arrhythmia. A post hoc analysis of safety data from studies of heart failure used 1-day electrocardiograms as surrogates of clinically meaningful arrhythmia and showed no differences versus the placebo [8]. This finding, together with reports of decreased mortality with levosimendan therapy in heart failure, suggests an absence of electrophysiological effects even if this remains discussed [6]. Duchenne muscular dystrophy shares with many muscular dystrophies a number of direct effects on the myocardium, which manifest as heart failure, conduction disturbances, atrial and ventricular arrhythmias, and sudden death. Given the 35-day interval between levosimendan discontinuation in our patient, the 1-week half-life of the active metabolites, and known cardiac complications of Duchenne muscular dystrophy, we believe the fatal arrhythmia in our patient was unrelated to the use of levosimendan.

\section{Conclusion}

Despite the administration of only one-third of the standard total dose, levosimendan associated to dobutamine and norepinephrine substantially improved both the left ventricular ejection fraction and the uniformity of heart wall motion in our patient with Duchenne muscular dystrophy-associated cardiomyopathy. This outcome suggests 
that levosimendan could be an adjunct treatment of severe exacerbations of Duchenne muscular dystrophy-associated heart failure refractory to maximal-dose vasopressor therapy.

\section{Compliance with Ethical Standards}

Conflict of interest The authors declare that they have no conflict of interest.

Ethical Approval The authors assert that all procedures contributing to this work comply with the ethical standard of the relevant national guidelines on human experimentation (1988 Huriet Law and 2004 Public Health Policy Law) and with the Helsinki Declaration of 1975, as revised in 2008, and has been approved by the institutional committee Comité de Protection des Personnes Ile-de-France XI, Saint-Germain en Lay, France.

\section{References}

1. Nigro G, Comi LI, Politano L, Bain RJ (1990) The incidence and evolution of cardiomyopathy in Duchenne muscular dystrophy. Int J Cardiol 26:271-277

2. Hasenfuss G, Pieske B, Castell M, Kretschmann B, Maier LS, Just $\mathrm{H}$ (1998) Influence of the novel inotropic agent levosimendan on isometric tension and calcium cycling in failing human myocardium. Circulation 98:2141-2147

3. Lunghetti S, Palmerini E, Urselli R et al (2011) Effects of levosimendan without loading dose on systolic and diastolic function in patients with end-stage heart failure. Cardiol J 18:532-537

4. Morelli A, De Castro S, Teboul JL et al (2005) Effects of levosimendan on systemic and regional hemodynamics in septic myocardial depression. Intensive Care Med 31:638-644

5. Follath F, Cleland JG, Just H et al (2002) Efficacy and safety of intravenous levosimendan compared with dobutamine in severe low-output heart failure (the LIDO study): a randomised doubleblind trial. Lancet 360:196-202

6. Flevari P, Parissis JT, Leftheriotis D, Panou F, Kourea K, Kremastinos DT (2006) Effect of levosimendan on ventricular arrhythmias and prognostic autonomic indexes in patients with decompensated advanced heart failure secondary to ischemic or dilated cardiomyopathy. Am J Cardiol 98(12):1641-1645

7. Antila S, Sundberg S, Lehtonen LA (2007) Clinical pharmacology of levosimendan. Clin Pharmacokinet 2007;46(7):535-552 (Review)

8. Lilleberg J, Ylönen V, Lehtonen L, Toivonen L (2004) The calcium sensitizer levosimendan and cardiac arrhythmias: an analysis of the safety database of heart failure treatment studies. Scand Cardiovasc J 38(2):80-84

Publisher's Note Springer Nature remains neutral with regard to jurisdictional claims in published maps and institutional affiliations. 